\title{
Can histologically normal epileptogenic zone share common electrophysiological phenotypes with focal cortical dysplasia? SEEG-based study in MRI-negative epileptic patients
}

Stanislas Lagarde*11, Julia Scholly*1,2, Irina Popa², Maria Paola Valenti-Hirsch², Agnès Trebuchon1, Aileen McGonigal ${ }^{1}$, Mathieu Milh³ ${ }^{3}$ Anke M. Staack4, Béatrice Lannes ${ }^{5}$, Benoît Lhermitte ${ }^{5}$, François Proust $^{2}$, Mustapha Benmekhbi², Didier Scavarda ${ }^{6}$, Romain Carron ${ }^{7}$, Dominique FigarellaBranger ${ }^{8}$, Edouard Hirsch ${ }^{2}$, Fabrice Bartolomei ${ }^{1}$.

1 Aix Marseille Univ, APHM, INSERM, INS, Inst Neurosci Syst, Timone Hospital, Epileptology Department, Marseille, France;

2 Medical and Surgical Epilepsy Unit, Hautepierre Hospital, Strasbourg, France;

${ }^{3}$ APHM, Timone Hospital, Paediatric Neurology, Marseille, France;

${ }^{4}$ Kork Epilepsy Center, Kehl-Kork, Germany;

${ }^{5}$ Department of Pathology, Hautepierre Hospital, Strasbourg, France;

${ }^{6}$ APHM, Timone Hospital, Pediatric Neurosurgery, Marseille, France;

${ }^{7}$ Aix Marseille Univ, APHM, INSERM, INS, Inst Neurosci Syst, Timone Hospital, Functional and Stereotactic Neurosurgery, Marseille, France;

8 Aix-Marseille Univ, APHM, Institut de Neurophysiopathologie, Timone Hospital, Anatomopathology Department, Marseille, France.

${ }^{*}$ These authors contributed equally to this work

\section{Corresponding author:}

Pr Fabrice Bartolomei, MD, PhD

Service d'Epileptologie

Hôpital Timone - 264 Rue St Pierre 13005-Marseille, France

Tel:+33491385833 Fax :+33491385826

Email: fabrice.bartolomei@ap-hm.fr

Key words: Epilepsy; MRI-negative; Focal cortical dysplasia; SEEG; Seizure-onset; Epileptogenic zone 


\begin{abstract}
Objective: We aimed to assess stereoelectroencephalography (SEEG) seizure-onset and interictal patterns associated with MRI-negative epilepsy and investigate their possible links with histology, extent of the epileptogenic zone (EZ) and surgical outcome.
\end{abstract}

Methods: We retrospectively analysed a cohort of 59 consecutive MRI-negative surgical candidates, who underwent SEEG recordings followed by cortectomy between 2000 and 2016.

Results: Most of the eight distinct seizure-onset patterns could be encountered both in confirmed focal cortical dysplasia (FCD) and in histologically non-specific or normal cases. We found strong correlation $(\mathrm{p}=0.008)$ between seizure-onset pattern and histology for: (1) slow-wave/DC-shift prior to low voltage fast activity (LVFA), associated with normal/non-specific histology, and (2) bursts of polyspikes prior to LVFA, exclusively observed in FCD.

Three interictal patterns were identified: periodic slow-wave/gamma burst, subcontinuous rhythmic spiking and irregular spikes. Both "periodic" patterns were more frequent in but not specific to FCD.

Surgical outcome depended on the EZ complete removal, regardless electrophysiological features.

\title{
Conclusions
}

Histologically normal and FCD-associated epileptogenic zones share distinct interictal and ictal electrophysiological phenotypes, with common patterns between FCD subtypes and between dysplastic and apparently normal brain.

Significance: Some specific seizure-onset patterns seem to be predictive of the underlying histology and may help to detect an MRI-invisible FCD. 


\section{HIGHLIGHTS}

- Epileptogenic Zones with normal histology \& FCD share common electrophysiological phenotypes

- Seizure-onset patterns are better predictors of the histological substrate than interictal patterns

- Burst of polyspikes and DC-shift prior to LVFA are respectively predictive of FCD \& normal histology 


\section{Introduction}

Focal pharmacoresistant epilepsy with normal or non-contributory magnetic resonance imaging (MRI) constitutes one of the main indications for presurgical evaluation with stereoelectroencephalography (SEEG)[1, 2]. The concept of MRI-negative focal epilepsy continues to evolve with advances in neuroimaging that allow better detection of subtle lesions [3, 4], as well as with the employment of novel approaches of EEG signal analysis for identifying the anatomic distribution of the epileptogenic process. Resective surgery is an increasingly successful treatment option, with 44 to $79 \%$ of drug-resistant MRInegative patients becoming seizure-free [5-9], especially using SEEG evaluation and tailored resection[10]. In varying proportions of these resections, a well characterized and systematically suspected developmental pathology such as focal cortical dysplasia (FCD) type II, more subtle FCD type I, or a less specific abnormality such as mild malformation of cortical development (mMCD) may ultimately be confirmed on neuropathological examination [11-14]. However, no specific microscopic lesion is identified in around 8\% of MRI-negative cases, with considerable variations (2 to 26\%) between reported series $[11,15,16]$.

Although the presence of a distinct epileptogenic lesion on histopathology has been strongly associated with a more favourable postsurgical outcome [5, 17-20], the extension of the epileptogenic zone (EZ), as well as completeness of the EZ resection represent independent predictive variables influencing long-term seizure outcome, both in lesion-associated and in non-lesional cases [8, 13, 21, 22]. Optimal definition of the EZ requires SEEG recordings in the majority of MRI-negative cases. One advantage of the SEEG method in this scenario is the ability to detect small bottom-of-sulcus dysplasia type II[23]. Indeed it has been suggested that there are characteristic SEEG electrophysiological signatures that suggest a high likelihood of this underlying 
aetiology $[24,25]$. On the other hand, the intrinsic epileptogenicity per se of a detected lesion does not necessarily indicate a focal, "lesional" organization of the epileptogenic zone [26], the definition of which remains primarily based on anatomo-electro-clinical correlations.

Several studies have suggested the relevance of intracranial seizure-onset patterns (SOP) to guide successful surgical resections and estimate potential surgical outcome [27-30]. In addition to the above-mentioned interictal and ictal electrophysiological signatures of FCD type II [24, 25], the Marseille group recently identified six distinct SEEG seizure-onset patterns in FCD-associated focal seizures, which varied according to histologic subtypes [31]. However, the predictive value of observing such patterns in a mixed group of patients with MRI-negative epilepsy has not yet been established.

We aimed to assess SEEG electrophysiological patterns associated with MRI-negative epilepsy and to investigate possible links with histological substrate, the extension of the EZ, and surgical outcome.

\section{Methods}

\subsection{Patient selection}

Among all patients (children and adults) who underwent stereotactic intracerebral EEG (SEEG) recordings followed by cortectomy in our departments (Marseille and Strasbourg epilepsy centres) from 2000 to 2016, we retrospectively analysed all consecutive patients with preoperative normal or non-contributory brain MRI, and available histology, and at least 12 months of follow-up after epilepsy surgery. All patients had detailed evaluation including medical history, neurologic examination, fluorodeoxyglucose positron emission tomography (FDG-PET) and pre-surgical epilepsy protocol MRI. MRI examinations were performed using 1.5T scanners prior to 2006 (18 
patients) and 3T scanners from 2006 onwards (41 patients). The imaging protocol included at least: 3D T1-weighted gradient-recalled-echo with gadolinium, 3D T1weighted inversion-recovery axial and coronal fluid-attenuated inversion recovery (FLAIR), axial T2 and axial T2 gradient-echo. For 3D T1, voxel size was $1 \mathrm{~mm}$. For T2 and FLAIR, slice thickness was $3 \mathrm{~mm}$. MRI was interpreted by experienced neuroradiologists as well as been reviewed by the epilepsy surgery team, taking into account the electroclinical and FDG-PET data. In this study, all patients required SEEG exploration after non-invasive investigations, as a part of routine clinical management. SEEG was indicated to localize the epileptogenic zone and to precisely determine its relations with eloquent areas. The placement of electrodes was selected individually for each patient, based on non-invasive investigations (semiology, interictal and ictal scalp-EEG, interictal PET hypometabolism) providing hypotheses about the localization of the EZ. Postoperative seizure outcome was assessed at the latest available follow-up according to the Engel classification [32].

The institutional review board of the French Institute of Health (IRB15226) approved this study.

\subsection{SEEG recordings}

Recordings were performed using intracerebral multiple contact electrodes (10-15 contacts with length $2 \mathrm{~mm}$, diameter $0.8 \mathrm{~mm}$, and $1.5 \mathrm{~mm}$ apart). Signals were recorded on a 128 or 256-channel Natus system and sampled at 256, 512, or 1,024 Hz, depending on the period of recording, and recorded on a hard disk (16 bits/ sample) using no digital filter. Two hardware filters were present in the acquisition procedure: a highpass filter (cut-off frequency equal to $0.16 \mathrm{~Hz}$ at $-3 \mathrm{~dB}$ ), and an anti-aliasing low-pass filter (cut-off frequency equal to $97 \mathrm{~Hz}$ at $256 \mathrm{~Hz}, 170 \mathrm{~Hz}$ at $512 \mathrm{~Hz}$, or $340 \mathrm{~Hz}$ at 1,024 $\mathrm{Hz}$ ). 


\subsection{Signal analysis}

Seizure onset was analysed both in terms of seizure-onset pattern (SOP) and its dynamics, and in terms of spatial extension[33]. Seizure onset was defined as the first change of SEEG signal within the context of a sustained rhythmic discharge and subsequent appearance of clinical signs. Seizures in which clinical manifestations preceded electrographic onset were excluded from the analysis.

The anatomic definition of the epileptogenic zone (EZ), defined as the brain regions primarily involved in seizure genesis, was performed by visual and quantitative signal analysis according to the French guidelines on stereoelectroencephalography[2]. The quantitative analysis was performed using the epileptogenicity index (EI) [34]. The EI combines analyses of both spectral and temporal features of SEEG signals, respectively, related to the propensity of a brain area to generate fast discharges and to the earliness of involvement of this area in the seizure. EI values were computed and averaged for all recorded seizures. The EZ was defined as the structure(s) with an EI value superior or equal to 0.4 [26], or by visual analysis (17 of 59 patients) in cases with seizures starting by patterns of lower frequencies (alpha and theta range) .

We analysed the seizure-onset patterns as well as the interictal patterns (IIP) exhibited by structures within the EZ. We used the same methodology as in our previous report to define the seizure-onset patterns [31]. Briefly, the SOP and IIP were assessed by three examiners (SL, JS or IP), blind to patients' clinical data. The electrographic patterns were described using visual analysis without software filters, and spectral analysis using AnyWave software [35]. The discrepancies were solved through consensus after discussion with a senior epileptologist (FB). 
Interictal activity (background, spikes, spike and wave complexes, slow activities, fast activities) was assessed on the electrodes that belonged to the EZ during a $10 \mathrm{~min}$ period of resting state in waking and during 10 min of NREM sleep. Sleep recordings were chosen because interictal epileptic activity and in particular, pseudoperiodic patterns pathognomonic of FCD type II are known to be activated in NREM sleep [24, 36, 37]. Interictal SEEG samples were selected at least $24 \mathrm{~h}$ after the SEEG implantation and with $24 \mathrm{~h}$ of distance from a seizure.

The extension of the EZ was evaluated as the number of distinct anatomo-functional brain regions (further referred to as "sub-lobes") involved, i.e. including at least one structure with high epileptogenicity index $(\geq 0.4)$ or defined as such by visual analysis for the cases with lower frequency patterns of seizure onset. We distinguished the following sub-lobes, based on the available depth electrode sampling: mesial temporal, lateral temporal, insula, orbito-frontal, mesial prefrontal, lateral prefrontal, premotor, central (pre- and post-central), opercular (frontal and rolandic), mesial parietal, lateral parietal, mesial occipital, lateral occipital.

The topographic organization of the EZ was defined as temporal, temporal plus (EI maximum within temporal lobe but also area with EI $>0.4$ outside temporal lobe such as insula, frontal or occipital lobe), operculo-insular (opercular, insular or operculoinsular), frontal , frontal plus (EI maximum within frontal lobe but also area with EI $>0.4$ outside frontal lobe such as temporal, insula or parietal), parietal, occipital.

\subsection{Pathological examination}

Analysis of resected specimens was performed en bloc by an expert neuropathologist from each contributing centre (BL, BLh or DFB) according to the international recommendation of the ILAE consensus Task Force [38] . Histopathological diagnosis was established according to the ILAE classification [39]. Three groups of patients were 
established for further analysis: patients with FCD type I, patients with FCD type II, and patients with "normal" histology. The latter group comprised the cases with no abnormalities or nonspecific reactive gliosis, or with the presence of isolated heterotopic neurons in deep subcortical white matter location, defined as mild malformation of cortical development type II $[39,40]$, since the pathologic value of this aspect, frequently encountered in non-epileptic patients' specimens, has not been clearly established [41].

The correspondence between the anatomical targets of the recording contacts within the SEEG-defined EZ and the histologically identified resected structures was checked using 3D CT/MRI data fusion of post-operative MRI and post-implantation cranial CT with electrodes.

\subsection{Statistical Analysis}

For statistical analysis, we defined one SOP per patient since the majority of patients exhibited the same ictal pattern across recorded seizures. For patients with more than one distinct SOP (only two cases), we used the most prevalent pattern as the representative SOP. Two rare SOP (delta-brush, and sharp beta) that were found only in one patient each, were excluded from statistical analysis. Because of low patient numbers in certain categories and non-normal distribution of data, we used nonparametric statistical tests: Fisher's exact test for nominal variables and Kruskal-Wallis test for numerical data. We applied Bonferroni correction for multiple comparisons. A pvalue $<0.05$ was considered as significant. Statistical tests were performed with the software Statistical Package for the Social Sciences (SPSS) for Mac, version 22.

\section{RESULTS}




\subsection{Patients' features}

Fifty-nine patients (34 women and 25 men) were included. Mean age at epilepsy onset was $12.6 \pm 8.1$, mean age at SEEG was $30.4 \pm 12$, and mean epilepsy duration was $17.8 \pm$ 10 years. We found a family history of epilepsy in 20 patients (33.9\%). The epileptogenic zone comprised a mean of 1.7 sub-lobes (range: 1-4). Histopathological examination revealed FCD type I in eight (13.6\%), FCD type II in ten (16.9\%) including presence of balloon cells in six (10,2\%), mMCD type II in four (6.8\%), and no abnormality or nonspecific reactive gliosis in 37 patients $(62.7 \%)$. The clinical data with respect to histological phenotypes are detailed in Table 1.

\subsection{Seizure onset patterns}

We identified eight distinct seizure-onset patterns (Fig.1, for definition, see supplementary data): slow-wave/DC shift prior to low voltage fast activity (LVFA) in 18 patients (30.5\%), LVFA in 11 (18.6\%), theta/alpha sharp in ten (16.9\%), pre-ictal spiking prior to LVFA in nine (15.3\%), rhythmic slow spikes in six (10.2\%), burst of poly-spikes prior to LVFA in three (5.1\%), delta-brush and beta sharp in two patients (1.7\%). The latter two patterns, each represented by a single case, were excluded from further analysis as previously indicated. SOPs including LVFA were the most prevalent in the FCD type II (80\%) and histologically normal groups (76\%), whereas they were encountered in only $50 \%$ of patients with FCD type I. Regarding the association between SOP and histological type, we found more bursts of polyspikes prior to LVFA in patients with FCD II, more rhythmic slow spikes in patients with FCD I and a significantly higher proportion of slow-wave/DC shift in patients with histologically normal EZ compared to the FCD groups ( $p=0,008$, with Bonferroni; Fig. 3A). Two particular SOP proved to be highly indicative of the histological type: (1) bursts of polyspikes prior to LVFA were only found in patients with FCD (Positive Predictive Value [PPV] = 100\%; Negative 
Predictive Value $[N P V]=72.2 \%$; Sensitivity $=16.7 \%$ and Specificity $=100 \%)$, and $(2)$ slow-wave/DC shift prior to LVFA was mostly found in normal histology and not found in FCD I (PPV = 88.9\%; NPV= 41\%, Sensitivity = 41\%, Specificity = 88.9\%).

No significant association was demonstrated between SOP and other clinical variables (gender, family history of epilepsy, age at SEEG, epilepsy duration, presence of balloon cells, topographic organization of the EZ and the extension of the EZ).

\subsection{Interictal patterns}

We identified three distinct interictal patterns (Fig. 2):

(1) Slow-wave/gamma burst characterized by periodic bursts of low-voltage gamma-oscillations $(40-55 \mathrm{~Hz})$, superimposed on a slow wave or spike-slow wave complex $(0.5-1 \mathrm{~Hz})$, interspaced with poor background activity;

(2) Sub-continuous rhythmic spiking characterized by periodic or pseudoperiodic rhythmic spikes or polyspikes, activated during NREM sleep, with poor or absent background activity;

(3) Irregular spikes, characterized by irregular spike, spike-wave or polyspike-wave activity, which could be activated during NREM sleep, with preserved background activity or background slowing.

We found irregular spikes in 35 patients (59.3\%), slow-wave with gamma burst in 13 (22\%) and sub-continuous rhythmic spiking in 11 patients (18.6\%). There was higher prevalence of irregular spikes in the histologically normal EZ but with limited predictive performances $(\mathrm{PPV}=77.1 \%$; NPV= 41.7\%, Sensitivity $=65.8 \%$, Specificity $=55.6 \%)$. Both "periodic" IIP, slow-wave/gamma-burst and sub-continuous rhythmic spiking, were more prevalent in dysplastic EZ with good specificity but low sensitivity (slowwave/gamma-burst: PPV = 30.7\%; NPV = 69.6\%, Sensitivity $=22.2 \%$, Specificity $=78 \%$; sub-continuous rhythmic spiking: PPV $=54.5 \%$;PV $=75 \%$, Sensitivity $=33.3 \%$, 
Specificity $=87.8 \%$ ). Interestingly, slow-wave/gamma-burst was not observed in FCD I, whereas sub-continuous rhythmic spiking occurred in both FCD I and FCD II groups as well as in patients with histologically normal EZ (Fig.3B).

However, there was no statistically significant association between IIP and histology $(p=0.1)$. IIP were significantly associated with epilepsy duration $(p=0.02)$ that was longer in patients with sub-continuous rhythmic spiking (supplementary figure). No significant association was demonstrated between IIP and others clinical variables (gender, family history of epilepsy, age at SEEG, presence of balloon cells, topographic organization of the EZ and the extension of the EZ).

\subsection{Association between interictal and seizure onset patterns}

There was no statistically significant association between the seizure-onset and interictal patterns $(\mathrm{p}=0.26)$.

\subsection{Association with post-surgical prognosis}

The mean follow-up after surgery was $5.4 \pm 3.4$ years. Thirty-five patients $(59.3 \%)$ were seizure free at last follow-up (Engel class I); eight patients were "almost seizure free" (13.6\%, Engel II), worthwhile improvement was achieved in seven (11.9\%, Engel III) and no improvement occurred in nine cases (15.3\%, Engel IV). No significant association was found between seizure-onset or interictal patterns and post-surgical seizure prognosis $(p=0.7$ and $p=0.18$ respectively, Fig. 4A \& 4B). However, favourable surgical outcome was positively associated with the complete removal of the EZ and negatively associated with presence of family history of epilepsy $(\mathrm{p}<0.001$ and $\mathrm{p}=0.02$ respectively, Fig. 4C \& 4D). There was also a significant association between post-surgical prognosis and the EZ topography $(\mathrm{p}=0.025)$ with poorer prognosis for temporal plus epilepsies. No significant association was demonstrated between prognosis and other clinical variables (including presence or not of a FCD, histological subtype, gender, epilepsy 
duration, age at SEEG or EZ extension).

\section{Discussion}

In this two-centre study, we compared the SEEG-derived electrophysiological phenotypes of epileptogenic zones associated with confirmed cortical dysplasia with those associated with cortex that was histologically normal or showed non-specific changes, in a longitudinal surgical cohort of patients with MRI-negative focal epilepsy. We particularly evaluated whether some seizure-onset or interictal patterns were predictive of underlying histological substrate, extension of the epileptogenic zone, or postsurgical seizure outcome.

\subsection{Seizure-onset patterns: key findings}

We confirmed that essentially six distinct SEEG-based seizure-onset patterns, described in our previous study [31], are likely to be encountered in MRI-negative FCD type II and type I. Furthermore, we have shown for the first time that most of these patterns can also be found in EZ associated with cortex showing no or non-specific histopathological changes. Two additional SOP (sharp beta and delta-brush) were observed in patients with normal histology, which were however extremely rare in the present series, in line with the existing literature $[30,42]$.

Patterns including low voltage fast oscillations at seizure onset were clearly overrepresented (>70\%) in FCD II and in histologically normal EZ, whereas SOP with slower rhythmic activity were more often observed in FCD I where they represented as much as half of cases. The latter findings support the hypothesis that such "slow" patterns may correspond to a particular mode of seizure initiation in these developmental malformations [31].

One of the key findings of our study is the strong association between the SEEG pattern 
and the underlying histological substrate, demonstrated for two distinct SOP. The first, bursts of polyspikes prior to LVFA, was exclusively observed in patients with FCD. Despite limitations due to the lower FCD prevalence in the present MRI-negative series, and in agreement with the previous study on FCD [31], our data suggest that this SOP might be highly predictive of underlying FCD but not of FCD type. This finding is of particular importance. Since intracranial SOP have been shown to correlate well with scalp SOP in cases of superficial FCD [43], the observation of similar pattern on scalp EEG might then be highly suggestive of superficial FCD.

The second, slow-wave/DC-shift pattern albeit the most prevalent in the whole cohort, was highly indicative of histologically normal EZ. However, its specificity might be overestimated, when considering the above-mentioned limitation.

\subsection{Interictal patterns: key findings}

The electrophysiological expression of MRI-negative EZ during the interictal state was generally characterized either by irregular spikes or by one of two distinct periodic patterns (slow-wave/gamma burst or sub-continuous rhythmic spiking). Although both periodic IIP were more prevalent in dysplastic EZ, they were not predictive of any

particular histological substrate. Noticeably, sub-continuous rhythmic spiking, classically described as pathognomonic of FCD type II, in particular in the presence of balloon cells (FCD IIb) [24, 25], was observed equally often in both FCD types and was also found in $12 \%$ of cases with histologically normal EZ in our series. Finally, this periodic interictal pattern was associated with longer duration of epilepsy in the present study.

\subsection{Significance and pathophysiological considerations}


Taken together, our findings highlight two points in epileptic patients with normal MRI: 1) some SOP and IIP are suggestive of dysplastic EZ with good specificity but poor sensitivity; 2) SOP seem to be better predictors of the histological substrate than IIP.

The association between SOP and histological substrate is probably related to different pathophysiological mechanisms reflecting the fact that FCD subtypes have specific and distinct alterations of neuronal populations, e.g. interneurons [44]. There is growing evidence from human and experimental studies that GABAergic interneurons play a pivotal role both in interictal [45] and ictal pattern genesis [46, 47]. The observed diversity of electrographic patterns might mirror different functional or morphofunctional alterations of GABAregic circuits, undetectable in a routine diagnostic setting [48]. However, not only cellular (glial, neuronal), but also molecular or genetic alterations [49] might influence the electrophysiological phenotype during the interictal and ictal state in true lesion-negative focal epilepsy.

Finally, both in lesion-associated and non-lesional cases, changes in functional connectivity within the epileptogenic zone network are discussed as possible mechanism underlying the transition from interictal to ictal period [50]. The question whether such connectivity alterations, either locally driven by an intrinsically epileptogenic lesion, or immediately involving more distributed, cortico-cortical or cortico-subcortical circuits, might determine distinct electrophysiological phenotypes, remains to be elucidated in future studies.

\subsection{Association with postsurgical seizure prognosis}

Regarding prognosis, we did not confirm the results of previous studies [28, 29, 42] suggesting that SOP including LVFA might be predictive of more favourable postsurgical outcome. Our data rather indicate that ictal patterns with lower frequency activities at 
seizure onset are not necessarily associated with poorer prognosis. In the present study of the MRI-negative patient cohort, no significant association was found between individual electrophysiological patterns and postsurgical outcome (59.3\% seizure-free) or extension of the EZ These findings underline the fact that, although some electrophysiological patterns are evocative of specific histological lesions, this does not guarantee focal, "lesional" organization of the EZ. Finally, our study confirms that complete removal of the EZ is, by far, the most important prognostic factor [31]. However, potential benefit of resective surgery might be reduced if family history is positive for epilepsy.

The presence of family history of epilepsy in about one third of our patients, including those with FCD, corroborates the hypothesis of genetic aetiology as possible common background in some cases with MRI-negative focal epilepsy [51]. This could explain the observed common electrophysiological phenotypes between the FCD I and II, and between the dysplastic and histologically normal EZ. In this respect, it might be pertinent to search for direct correlations between genotype and electrophysiological phenotype by performing gene panels or exome sequencing on resected tissue. Demonstrating somatic mutations e.g. mTOR might, in the future, influence treatment strategies by offering personalized therapeutic approaches to these patients [52].

\subsection{Study limitations}

Our study has some limitations, including the relative under-representation of FCD cases. The detection of lesions in the resected material might be influenced by difficulties of per operative specimen sampling as well as by an incomplete resection of the presumably epileptogenic cortex due to the overlap with functional areas, so that some false-negative cases of small FCD II, isolated or associated with FCD I, cannot be 
excluded. Further on, we acknowledge that some recently described entities such as mMCD, isolated or possibly associated with oligodendroglial hyperplasia, might be potentially over- or underdiagnosed, due to a lack of universally agreed pathological criteria $[17,46]$. Notwithstanding, as already mentioned above, the pathologic value of these conditions with regard to the focal epilepsies has not yet been clearly established. Finally, although the prevalence of histologically normal cases in our two-centre cohort was high enough (63\%) to control the latter limitation, our results could still be biased by retrospective design of the present study. Thus, the electrophysiological prognostic criteria in surgically remediable epilepsy with or without structural lesion require further evaluation in a prospective, multi-centre cohort.

\section{Conclusions}

Histologically normal and FCD-associated epileptogenic zones share distinct interictal and ictal electrophysiological phenotypes, with common patterns between FCD types I and II and between dysplastic and apparently normal brain. Some particular seizure onset patterns (slow-wave/DC-shift, burst of polyspikes prior to LVFA) seem to be highly predictive of the underlying histological substrate and may help to detect an MRIinvisible FCD. However, in the present study, no significant association was found between seizure-onset or interictal patterns and postsurgical outcome, which most strongly depended on the complete resection of the EZ and was negatively associated with family history of epilepsy. 


\section{ACKNOWLEGEMENTS}

We thank Prof. Henry Dufour and Prof. Jean-Claude Peragut (Marseille), Prof. Pierre Kehrli (died May 28, 2014) and Dr. Alexander Timofeev (Strasbourg) for surgical procedures in some of the selected patients.

We thank Prof. Patrick Chauvel, Prof Jean Régis, Prof. Martine Gavaret, Dr. Francesca Bonini, Dr. Lisa Vaugier, Dr. Sandrine Aubert, Dr. Geraldine Daquin, Dr. Constanza Dalvit, Dr. Nathalie Villeneuve and Dr. Anne Lepine (Marseille), Dr. Anne De Saint Martin, Dr. Clotilde Boulay, Dr Christel Dentel, Dr Charles Behr and Dr. Serge Chassagnon (Strasbourg) for the clinical management of some included patients.

\section{FUNDING}

This work has been carried out within the FHU EPINEXT with the support of the A*MIDEX project (ANR-11-IDEX-0001-02) funded by the "Investissements d'Avenir" French Governement program managed by the Agence Nationale de la Recherche (ANR) Part of this work was funded by a joint Agence Nationale de la Recherche (ANR) and Direction Génerale de l'Offre de Santé (DGOS) under grant "VIBRATIONS" ANR-13PRTS-0011-01.

Declarations of interest: The authors declare that they have no conflict of interest.

\section{REFERENCES}


1. Jayakar P, Gotman J, Harvey AS, et al (2016) Diagnostic utility of invasive EEG for epilepsy surgery: Indications, modalities, and techniques. Epilepsia 57:17351747. https://doi.org/10.1111/epi.13515

2. Isnard J, Taussig D, Bartolomei F, et al (2017) French guidelines on stereoelectroencephalography (SEEG). Neurophysiol Clin. https://doi.org/10.1016/j.neucli.2017.11.005

3. Garbelli R, Milesi G, Medici V, et al (2012) Blurring in patients with temporal lobe epilepsy: Clinical, high-field imaging and ultrastructural study. Brain 135:23372349. https://doi.org/10.1093/brain/aws149

4. Zucca I, Milesi G, Medici V, et al (2016) Type II focal cortical dysplasia: Ex vivo 7T magnetic resonance imaging abnormalities and histopathological comparisons. Ann Neurol 79:42-58. https://doi.org/10.1002/ana.24541

5. Alarcón G, Valentín A, Watt C, et al (2006) Is it worth pursuing surgery for epilepsy in patients with normal neuroimaging? J Neurol Neurosurg Psychiatry 77:474-480. https://doi.org/10.1136/jnnp.2005.077289

6. Carne RP, O’Brien TJ, Kilpatrick CJ, et al (2004) MRI-negative PET-positive temporal lobe epilepsy: A distinct surgically remediable syndrome. Brain 127:2276-2285. https://doi.org/10.1093/brain/awh257

7. Kogias E, Klingler J-H, Urbach H, et al (2017) 3 Tesla MRI-negative focal epilepsies: Presurgical evaluation, postoperative outcome and predictive factors. https://doi.org/10.1016/j.clineuro.2017.10.038

8. Jayakar P, Dunoyer C, Dean P, et al (2008) Epilepsy surgery in patients with normal or nonfocal MRI scans: Integrative strategies offer long-term seizure relief. Epilepsia 49:758-764. https://doi.org/10.1111/j.1528-1167.2007.01428.x

9. McGonigal A, Bartolomei F, Régis J, et al (2007) Stereoelectroencephalography in presurgical assessment of MRI-negative epilepsy. Brain 130:3169-3183. https://doi.org/10.1093/brain/awm218

10. Thorsteinsdottir J, Vollmar C, Tonn J-C, et al (2019) Outcome after individualized stereoelectroencephalography (sEEG) implantation and navigated resection in patients with lesional and non-lesional focal epilepsy. J Neurol 266:910-920. https://doi.org/10.1007/s00415-019-09213-3

11. Blumcke I, Spreafico R, Haaker G, et al (2017) Histopathological Findings in Brain Tissue Obtained during Epilepsy Surgery. N Engl J Med 377:1648-1656. https://doi.org/10.1056/NEJMoa1703784

12. Seo JH, Noh BH, Lee JS, et al (2009) Outcome of surgical treatment in non-lesional intractable childhood epilepsy. Seizure 18:625-629.

https://doi.org/10.1016/j.seizure.2009.07.007

13. Shi J, Lacuey N, Lhatoo S (2017) Surgical outcome of MRI-negative refractory extratemporal lobe epilepsy. Epilepsy Res 133:103-108. https://doi.org/10.1016/j.eplepsyres.2017.04.010

14. Wang ZI, Alexopoulos A V., Jones SE, et al (2013) The pathology of magneticresonance-imaging-negative epilepsy. Mod Pathol 26:1051-1058. https://doi.org/10.1038/modpathol.2013.52

15. De Tisi J, Bell GS, Peacock JL, et al (2011) The long-term outcome of adult epilepsy surgery, patterns of seizure remission, and relapse: A cohort study. Lancet 378:1388-1395. https://doi.org/10.1016/S0140-6736(11)60890-8

16. Schurr J, Coras R, Rössler K, et al (2017) Mild Malformation of Cortical Development with Oligodendroglial Hyperplasia in Frontal Lobe Epilepsy: A New 
Clinico-Pathological Entity. Brain Pathol 27:26-35.

https://doi.org/10.1111/bpa.12347

17. Blume WT, Ganapathy GR, Munoz D, Lee DH (2004) Indices of Resective Surgery

Effectiveness for Intractable Nonlesional Focal Epilepsy. Epilepsia 45:46-53.

https://doi.org/10.1111/j.0013-9580.2004.11203.x

18. Lee SK, Lee SY, Kim KK, et al (2005) Surgical outcome and prognostic factors of cryptogenic neocortical epilepsy. Ann Neurol 58:525-532.

https://doi.org/10.1002/ana.20569

19. Téllez-Zenteno JF, Ronquillo LH, Moien-Afshari F, Wiebe S (2010) Surgical outcomes in lesional and non-lesional epilepsy: A systematic review and metaanalysis. Epilepsy Res 89:310-318.

https://doi.org/10.1016/j.eplepsyres.2010.02.007

20. Chapman K, Wyllie E, Najm I, et al (2005) Seizure outcome after epilepsy surgery in patients with normal preoperative MRI. J Neurol Neurosurg Psychiatry 76:7103. https://doi.org/10.1136/jnnp.2003.026757

21. Fauser S, Essang C, Altenmüller D-M, et al (2015) Long-term seizure outcome in 211 patients with focal cortical dysplasia. Epilepsia 56:66-76.

https://doi.org/10.1111/epi.12876

22. McIntosh AM, Averill CA, Kalnins RM, et al (2012) Long-term seizure outcome and risk factors for recurrence after extratemporal epilepsy surgery. Epilepsia 53:970-978. https://doi.org/10.1111/j.1528-1167.2012.03430.x

23. Chassoux F, Landré E, Mellerio C, et al (2012) Type II focal cortical dysplasia: Electroclinical phenotype and surgical outcome related to imaging. Epilepsia 53:349-358. https://doi.org/10.1111/j.1528-1167.2011.03363.x

24. Chassoux F, Devaux B, Landre E, et al (2000) Stereoelectroencephalography in focal cortical dysplasia: A 3D approach to delineating the dysplastic cortex. Brain 123:1733-1751. https://doi.org/10.1093/brain/123.8.1733

25. Tassi L, Colombo N, Garbelli R, et al (2002) Focal cortical dysplasia: neuropathological subtypes, EEG, neuroimaging and surgical outcome. Brain 125:1719-1732. https://doi.org/10.1093/brain/awf175

26. Aubert S, Wendling F, Regis J, et al (2009) Local and remote epileptogenicity in focal cortical dysplasias and neurodevelopmental tumours. Brain 132:3072-3086. https://doi.org/10.1093/brain/awp242

27. Holtkamp M, Sharan A, Sperling MR (2012) Intracranial EEG in predicting surgical outcome in frontal lobe epilepsy. Epilepsia 53:1739-1745. https://doi.org/10.1111/j.1528-1167.2012.03600.x

28. Jiménez-Jiménez D, Nekkare R, Flores L, et al (2015) Prognostic value of intracranial seizure onset patterns for surgical outcome of the treatment of epilepsy. Clin Neurophysiol 126:257-267.

https://doi.org/10.1016/j.clinph.2014.06.005

29. Lee S-A, Spencer DD, Spencer SS (2000) Intracranial EEG Seizure-Onset Patterns in Neocortical Epilepsy. Epilepsia 1:297-307. https://doi.org/10.1111/j.15281157.2000.tb00159.x

30. Perucca P, Dubeau F, Gotman J (2014) Intracranial electroencephalographic seizure-onset patterns: Effect of underlying pathology. Brain 137:183-196. https://doi.org/10.1093/brain/awt299

31. Lagarde S, Bonini F, McGonigal A, et al (2016) Seizure-onset patterns in focal cortical dysplasia and neurodevelopmental tumors: Relationship with surgical prognosis and neuropathologic subtypes. Epilepsia 57:1426-1435. 
https://doi.org/10.1111/epi.13464

32. Engel J (1993) Update on surgical treatment of the epilepsies. Summary of the Second International Palm Desert Conference on the Surgical Treatment of the Epilepsies (1992). Neurology 43:1612-7.

https://doi.org/10.1212/WNL.43.8.1612

33. Bartolomei F, Nica A, Valenti-Hirsch MP, et al (2017) Interpretation of SEEG recordings. Neurophysiol Clin. https://doi.org/10.1016/j.neucli.2017.11.010

34. Bartolomei F, Chauvel P, Wendling F (2008) Epileptogenicity of brain structures in human temporal lobe epilepsy: A quantified study from intracerebral EEG. Brain 131:1818-1830. https://doi.org/10.1093/brain/awn111

35. Colombet B, Woodman M, Badier JM, Bénar CG (2015) AnyWave: A cross-platform and modular software for visualizing and processing electrophysiological signals. J Neurosci Methods 242:118-126. https://doi.org/10.1016/j.jneumeth.2015.01.017

36. Tassi L, Garbelli R, Colombo N, et al (2012) Electroclinical, MRI and surgical outcomes in 100 epileptic patients with type II FCD. Epileptic Disord 14:257-266. https://doi.org/10.1684/epd.2012.0525

37. Lambert I, Roehri N, Giusiano B, et al (2017) Brain regions and epileptogenicity influence epileptic interictal spike production and propagation during NREM sleep in comparison with wakefulness. Epilepsia 1-9. https://doi.org/10.1111/epi.13958

38. Blümcke I, Aronica E, Miyata H, et al (2016) International recommendation for a comprehensive neuropathologic workup of epilepsy surgery brain tissue: A consensus Task Force report from the ILAE Commission on Diagnostic Methods. Epilepsia 57:348-358. https://doi.org/10.1111/epi.13319

39. Blümcke I, Thom M, Aronica E, et al (2011) The clinicopathologic spectrum of focal cortical dysplasias: A consensus classification proposed by an ad hoc Task Force of the ILAE Diagnostic Methods Commission. Epilepsia 52:158-174. https://doi.org/10.1111/j.1528-1167.2010.02777.x

40. Palmini a, Najm I, Avanzini G, et al (2004) Terminology and classification of the cortical dysplasias. Neurology 62:S2-S8. https://doi.org/10.1212/01.WNL.0000114507.30388.7E

41. Liu JYW, Ellis M, Brooke-Ball H, et al (2014) High-throughput, automated quantification of white matter neurons in mild malformation of cortical development in epilepsy. Acta Neuropathol Commun 2:. https://doi.org/10.1186/2051-5960-2-72

42. Singh S, Sandy S, Wiebe S (2015) Ictal onset on intracranial EEG: Do we know it when we see it? State of the evidence. Epilepsia 56:1629-1638. https://doi.org/10.1111/epi.13120

43. Tanaka H, Khoo HM, Dubeau F, Gotman J (2018) Association between scalp and intracerebral electroencephalographic seizure-onset patterns: A study in different lesional pathological substrates. Epilepsia 59:420-430. https://doi.org/10.1111/epi.13979

44. Medici V, Rossini L, Deleo F, et al (2016) Different parvalbumin and GABA expression in human epileptogenic focal cortical dysplasia. Epilepsia 57:11091119. https://doi.org/10.1111/epi.13405

45. Muldoon SF, Villette V, Tressard T, et al (2015) GABAergic inhibition shapes interictal dynamics in awake epileptic mice. Brain. https://doi.org/10.1093/brain/awv227 
46. Shiri Z, Manseau F, Lévesque M, et al (2015) Interneuron activity leads to initiation of low-voltage fast-onset seizures. Ann Neurol 77:541-546. https://doi.org/10.1002/ana.24342

47. Avoli M, de Curtis M, Gnatkovsky V, et al (2016) Specific imbalance of excitatory/inhibitory signaling establishes seizure onset pattern in temporal lobe epilepsy. J Neurophysiol 115:3229-3237. https://doi.org/10.1152/jn.01128.2015

48. Cossart R, Tyzio R, Dinocourt C, et al (2001) Presynaptic kainate receptors that enhance the release of GABA on CA1 hippocampal interneurons. Neuron 29:497508. https://doi.org/10.1016/S0896-6273(01)00221-5

49. Kobow K, Blümcke I (2016) Epigenetics in epilepsy. Neurosci. Lett.

50. Bartolomei F, Wendling F, Régis J, et al (2004) Pre-ictal synchronicity in limbic networks of mesial temporal lobe epilepsy. Epilepsy Res 61:89-104. https://doi.org/10.1016/j.eplepsyres.2004.06.006

51. Baulac S, Ishida S, Marsan E, et al (2015) Familial focal epilepsy with focal cortical dysplasia due to DEPDC5 mutations. Ann Neurol 77:675-683. https://doi.org/10.1002/ana.24368

52. Guerrini R, Duchowny M, Jayakar P, et al (2015) Diagnostic methods and treatment options for focal cortical dysplasia. Epilepsia 56:1669-1686. https://doi.org/10.1111/epi.13200

\section{LEGENDS OF FIGURES AND TABLES}

Fig.1 The seizure-onset patterns according to the time frequency representation from SEEG trace. A: Low-voltage fast activity (LVFA); B: Preictal spiking followed by LVFA, C: Burst of polyspikes followed by LVFA; D: Slow wave or baseline shift followed by LVFA; E Rhythmic slow spikes; F: Theta/alpha sharp activity; G: beta sharp activity; H: delta-brush. The red asterisks mark the seizure onset.

\section{Fig. 2. The three interictal patterns according to the time-frequency} representation from SEEG trace. A: Slow-wave/gamma burst; B: Sub-continuous rhythmic spiking; C: Irregular spikes.

Fig. 3 (A) Prevalence of seizure onset patterns according to each 
histopathological phenotype; (B) Prevalence of interictal patterns according to each histopathological phenotype.

$\mathrm{FCD}=$ focal cortical dysplasia; LVFA= low voltage fast activity.

Fig. 4 Association between (A) the seizure-onset patterns, (B) the interictal patterns, (C) the family history of epilepsy, (D) completeness of the EZ resection and the postsurgical seizure outcome.

LVFA= low voltage fast activity; EZ= epileptogenic zone

Table 1: Detailed Clinical Data 